\title{
Kant et la "cosmogonie" du Beweisgrund de 1763: une nouvelle version de la Théorie du ciel?
}

\author{
Jean Seidengart*
}

Résumé: L'objectif de cet article est d'analyser et de comparer les différences significatives qui séparent la version abrégée de l'hypothèse cosmogonique de Kant qui figure dans le Beweisgrund de 1763 par rapport à celle que donnait l'édition complète de la Théorie du ciel de 1755. Une des principales différences entre ces deux exposés consiste dans le fait que Kant supprima dans la version de 1763 toute trace de son infinitisme cosmologique qui occupait pourtant une place éminente dans le texte de 1755. C'est le sens et les raisons de ce profond remaniement que cet article s'efforce de déterminer. Abstract: Here, the point is to analyse and to compare the main differences between the abbreviated version of Kant(scosmological hypothesis presented in The Only Possible Basis of Proof (1763) and the original one published in his Universal Natural History and Theory of the Heavens (1755). One of the most important differences between them is the fact that Kant withdrew, in 1763, all the statements concerning his cosmological infinitism, whereas in 1755 it was the chief feature of his cosmogony. This article wants to clear up the philosophical meaning and the reasons of this notable recasting.

KANT EST RESTÉ très attaché toute sa vie durant à l'hypothèse cosmologique qu'il publia en 1755 dans sa Théorie du ciel, ouvrage qui parut cependant sans nom d'auteur. Le faible impact que connut ce texte à l'époque, pour des raisons tout à fait externes, décida Kant, huit ans plus tard, à en revendiquer la paternité, et à annoncer au début du Bewveisgrund de 1763, non sans quelques réserves, qu'il allait reprendre de façon abrégée sa cosmologie de jeunesse, L'objet de la présente communication est précisément d'analyser et de comparer ces différences significatives qui séparent la version abrégée de l'édition complète de la Théorie du ciel.

Dans un premier temps, nous éluciderons le sens de ce retour de l'hypothèse cosmologique et son nouveau statut au sein du Beweisgrund. Ensuite, nous procéderons à une confrontation des deux versions de sa cosmologie afin d'en relever les principaux remaniements dans le texte de 1763. Enfin, dans un der-

* Professeur, Université de Reims, jean.seidengart@univ-reims.fr. 
nier temps, nous dégagerons les raisons qui poussèrent le Kant du Beweisgrund à supprimer toute trace de son infinitisme cosmologique qu'il avait pourtant mis au tout premier plan dans le texte de 1755.

\section{Le retour de l'hypothèse cosmologique dans le Beweisgrund et son nouveau statut}

\section{I.1. Kant revendique la paternité et la priorité de sa Théorie du ciel}

Comme on le sait, la Théorie du ciel de Kant avait été publiée sans nom d'auteur et sa parution était passée presque inaperçue en 1755 en raison de sa très faible diffusion, car son éditeur Johann Friedrich Petersen fit faillite et tout son stock d'exemplaires fut mis sous scellées. Dans son liv̄re de 1763 sur L'unique fondement possible d'une démonstration de l'existence de Dieu, Kant dut revenir sur le statut de la physico-théologie et profita de cette occasion pour présenter au public une nouvelle fois, mais sous une autre forme très abrégée, son hypothèse cosmogonique. C'est d'ailleurs dès I'Avant-propos du Beweisgrund que Kant précise en note et avoue expressément qu'il est l'auteur d'un livre dont "Le titre est Histoire générale de la nature et théorie du ciel" L'on sent une certaine amertume dans le ton de Kant qui déplore que la Théorie du ciel soit un écrit "peu connu"2 contrairement aux Lettres cosmologiques de Lambert ${ }^{3}$ dont il admire tant l'auteur manifestement. Kant fait remarquer au passage que sa Théorie du ciel avait vu le jour 6 ans avant l'hypothèse de Lambert, mais que, malgré sa priorité chronologique, il existe une profonde conformité entre ces deux cosmologies ${ }^{4}$

Or, il se trouve que le wolffien Sulzer, secrétaire de la classe de philosophie de l'Academie de Berlin, montra l'Unique fondement de Kant en 1764 à Lambert. Un an plus tard, Lambert, qui avait été touché par la note de Kant qui mentionne ses Lettres cosmologiques dans son Avant-propos, lui écrit:

1 Kant, L'Unique fondement possible..., 1763, AK II, p. 68; tr. fr. Zac, Paris, Pléiade, T. I, p. 321, note de l'auteur.

2 Ibidem.

3 J. H. Lambert, Cosmologische Briefe über die Einrichtung des Weltbaues, Augsburg, 1761, bey Eberhart Kletts Wittib.

4 Cf. Kant, L’Unique fondement possible..., 1763, AK II, p. 69; tr. fr. Zac, Paris, Pléiade, T. I, p. 322, note de l'auteur: "la conformité entre les pensées de cet homme ingénieux avec celles que j'ai exposées alors, s'accordant presque jusqu'aux moindres traits, renforce mon opinion que dans l'avenir cette hypothèse recevra des confirmations plus nombreuses" 
"Je puis vous assurer, cher Monsieur, que je né suis pas encore revenu de la mention que vous faites de ma cosmologie dans la préface à votre Unique fondement... Ce qui est dit dans les I.ettres cosmologiques [...] est à dater de 1749. [...] Je couchai sur une feuille l'idée que j'eus alors de considérer la Voie lactée comme un écliptique des étoiles fixes, et c'est tout ce que, $j$ 'avais produit en 1760 lorsque j'écrivis les Lettres...."5

Sans tomber dans une querelle de priorité, finalement assez stérile, Lambert tient seulernent à rétablir ses droits en cette matière et à préciser que ses vues concordent avec celles de Kant sans avoir été pour autant inspirées par la lecture de la Théorie du ciel. De son côté, Kant semble se réjouir à cette époque de cette profonde convergence de ses propres hypothèses cosmologiques avec celles de Lambert, qu'il n'hésite pas à désigner comme "le premier génie de l'Allemagne" 6 Toutefois, il faut signaler que lorsque Kant aura connu la gloire ainsi qu'une très grande cèlébrité après la publication de sa trilogie critique, il continuera toujours de revendiquer la priorité de ses vues cosmologiques sur celles de Lambert, mais il affirmera toutefois qu'une profonde divergence les sépare totalement. C'est ce que montre clairement la lettre de Kant à son ami mathématicien Johann Friedrich Gensichen du 19 Avril 1791:

"Afin d'accorder le crédit qui revient en propre à chacun de ceux qui ont contribué à l'histoire de l'astronomie, je souhaite que vous ajoutiez un appendice à votre ouvrage ${ }^{7}$ pour expliquer combien mes modestes conjectures personnelles diffèrent de celles des théoriciens ultérieurs.

1) La conception de la Voie lactée comme systèrne de soleils en mouvement analogue à notre système planétaire, je l'ai exposée six ans avant que Lambert ne publie une théorie similaire dans ses Lettres cosmologiques;

5 Lettre de Lambert à Kant du 15 novembre 1765, AK X, 50-51; Trad. fr. in Correspondance par I. Kant, Paris, Gallimard, 1991, p. 42-3.

6 Cf. lettre de Kant à Lambert du 31 décembre 1765, AK X, p. 54, Trad. in Pléiade, T. I, p. 595: "Je vous tiens pour le premier génie de l'Allemagne".

7 Cet ouvrage rassemblait des écrits de Herschel et de Kant, à savoir: W. Herschel, Sur la Constitution $d u$ ciel, trois mémoires traduits par G. M. Sommer, avec un précis authentique de l'Histoire générale de la nature et Théorie du Ciel de Kant, Königsberg, chez F. R. Nicolovius, 1791. Le texte de Kant figure de la p. 163 à 200. 
2) L'idée que les nébuleuses sont comparables à des Voies lactées lointaines n'a pas été hasardée par Lambert (quoique Erxleben le soutienne dans ses Anfangsgründe der Naturlebre page 540 , même dans sa nouvelle édition), puisqu'il les prenait pour des corps obscurs (au moins l'une d'entre elles), éclairés par des soleils voisins."8

Au lendemain de la publication de sa troisième Critique, Kant n'avait plus besoin de rechercher la caution scientifique de Lambert. Il convient donc de rester prudent au sujet des rapports entre Kant et Lambert en matière de cosmologie.

\section{I.2. La place de l'hypothèse cosmogonique dans 1'Unique fondement}

Certes, il est vrai que Kant accorde à la preuve a priori, c'est-à-dire ici sa preuve ontologique inversée, une prééminence indiscutable sur la preuve a posteriori, c'est-à-dire sur la preuve physico-théologique. Toutefois, Kant reconnaît expressément que la preuve ontothéologique inversée présente un caractère abstrait qui est rebutant pour la raison commune, tandis que la preuve a posteriori de la physico-théologie traditionnelle offre trois avantages particulièrement remarquables que n'a pas la première:

"la conviction qu'elle fait naître est extrêmement vive et attrayante et, par conséquent, facilement saisissable pour l'entendement commun, [...1 elle est plus spontanée qu'aucune autre, attendu que c'est indubitablement par elle que tout le monde

8 Lettre de Kant à Johann Friedrich Gensichen du 19 Avril 1791, in AK, XI, p. 252-253; et les KantStudien, II, 1897, p. 104 et ss. C'est nous qui traduisons. On trouve des précisions similaires dans la lettre de Kant à l'astronome J. E. Bode du 2 septembre 1790, AK XI, 203-4; trad. fr. in Correspondance, Paris, Gallimard, 1991, p. 431: "M. Herschel a découvert pour l'anneau de une rotation axiale de 10 h. $22^{\prime} 15^{\prime \prime}$ et ce à partir de la particule la plus proche du bord interne de cet anneau, cela pourrait venir confirmer ce que je supposais, il y a 35 ans, dans mon Histoire générale de la nature et Théorie du ciel. [...] La façon dont Monsieur Herschel représente les nébuleuses, c'est-à-dire comme un système en soi lui-même compris dans un système s'accorde à souhait avec ma façon de les exposer jadis in op. cit., p. 14-5; ce doit être un trou de mémoire de M. Erxleben que d'attribuer, dans sa physique, cette pensée à $\mathrm{M}$. Lambert qui serait le premier à l'avoir eue, alors que ses Lettres cosmologiques sont parues 6 ans après mes écrits, et que je ne parviens même pas, malgré toutes mes recherches, à y trouver ce type de représentation" 
commence; [...] elle procure une conception très intuitive de la sagesse suprême [...] qui, en remplissant l'âme, est éminemment propre à inspirer l'admiration, l'humilité et le respect." 9

Malheureusement, malgré tous ses avantages, la preuve physico-théologique a posteriori ne peut atteindre à une certitude matbématique, mais seulement à une certitude morale, c'est-à-dire une certitude qui suffit pour la conduite de la vie. Cependant, il est des cas où la certitude morale ne suffit pas, c'est lorsqu'il s'agit de "vaincre le scepticisme, [...] le plus éhonté"10. Maintenant, la question se pose de savoir pourquoi Kant éprouve le besoin de consacrer de très longs développements à la preuve physico-théologique si elle est philosophiquement inférieure à la preuve a priori et finalement insuffisante en elle-même?

La réponse est double: à savoir, d'une part, que les défauts de preuve physico-théologique traditionnelle peuvent être rectifiés, corrigés à partir d'une nouvelle "physicothéologie perfectionnée" et, d'autre part, que cette dernière peut servir de "complément" à la preuve a priori. Nous allons donc voir que la totalité de la deuxième des trois parties que comporte l'Unique fondement, est destinée à justifier et à établir la légitimité de l'hypothèse cosmogonique tirée de la Théorie $d u$ ciel dans la mesure où elle seule peut fournir une nouvelle forme, rénovée et perfectionnée, de la physico-théologie qui est conforme désormais à $<$ l'esprit de la vraie philosophie"11.

Tout d'abord, Kant définit la physico-théologie en général comme un procédé $<$ Art $>$, comme une méthode $<$ Metbode $>$ qui "remonte de la considération de la nature à la connaissance de Dieu"12 Plus précisément, cette considération de la nature recherche avant tout dans l'expérience des marques distinctives de régularité, de perfection, d'harmonie et de convenance dans l'ordre de la nature. Or, il existe trois manières d'envisager le statut de cet ordre naturel: la première fait sans cesse appel au miracle, mais elle a l'inconvénient d'“interrompre

9 Kant, L'Unique fondement possible..., 1763, AK II, p. 117; trad. fr. Pléiade, T. I, p. 381. Kant précise à la fin de son livre, IIIe. Partie, IV, AK II 161; trad. fr., p. 434: "Eu égard à l'utilité, cette preuve [physico-théologique] est préférable à toute autre preuve visant à plus de rigueur, et même à la mienne. [...] Des preuves de ce genre ont une réelle efficacité, et laissent une place plus grande à la faculté intuitive".

10 Op. cit., IIIe. Partie, IV, AK II 160; trad. fr., p. 433.

11 Kant, L’Unique fondement possible..., 1763, Iie. Partie, Vie. Considération, AK II, p. 136; trad. fr., Pléiade, T. I, p. 404.

12 Op. cit., IIe. Partie, Ve. Considération, AK II, p. 117; trad. fr., Pléiade, T. I, p. 380. 
à tout moment l'ordre de la nature"13; la seconde manière (d'allure wolffienne) fait ressortir le caractère contingent de l'ordre naturel pour conduire à Dieu, l'Etre nécessaire. Enfin, la troisième manière indique 1'“unité nécessaire perceptible dans la nature et l'ordre des choses [...] qui nous conduit à un principe suprême non seulement de cette existence, mais même de toute possibilité"14 Kant considère que la première manière est le propre des hommes à l'état sauvage, tandis que la seconde, qui est propre à toute âme bien née et civilisée, est conforme au bon sens et suffit à la vie vertueuse ${ }^{15}$ Seule la troisième manière relève de la philosophie proprement dite, c'est celle qu'il désigne comme la "méthode perfectionnée de la physico-théologie"16 tandis que la seconde était plutôt celle de "la physico-théologie ordinaire"17 Par conséquent, Kant ne veut pas rejeter la physico-théologie, mais seulement écarter ses deux premières formes qui stérilisent la réflexion ainsi que toute recherche, car elles rattachent toute perfection naturelle à un dessein contingent et particulier de la volonté divine. Bien au contraire conclut Kant:

"Il est louable d'appliquer ses efforts à améliorer cette méthode plutôt qu'à la combattre, à corriger ses défauts plutôt qu'à la mépriser à cause d'eux." 18

Kant doit encore fixer les nouvelles règles. de la méthode de cette physico-théologie perfectionnée, avant d'exposer les vues de son hypothèse cosmogonique.

\section{I.3. La Physico-théologie perfectionnée du Beweisgrun:}

Le newtonianisme de Kant a été profondément marqué par la signification toute particulière que Pierre Louis Moreau de Maupertuis lui a conférée, comme nous le verrons plus loin. En effet, Newton lui-même reconnaît à maintes reprises qu'il est nécessaire de faire appel aux causes finales et à l'intervention directe de Dieu en matière de cosmologie. Ainsi, Newton rappelle avec fer-

13 Ibidem, p. 116.

14 Ibidem.

15 Ibidem.

16 Op. cit., IIe. Partie, VIe. Considération, AK II, p. 123; trad. fr., Pléiade, T. I, p. 388.

17 Op. cit., IIe. Partie, Ve. Considération, AK II, p. 117; trad. fr., Pléiade, T. I, p. 381.

18 Op. cit., IIe. Partie, Ve. Considération, AK II, p. 123; trad. fr., Pléiade, T. I, p. 388. 
meté dans une lettre à Bentley que le système du Monde ne peut en aucune façon se passer de l'intervention divine:

"L'hypothèse qui dérive l'ordre du monde de l'action de principes mécaniques sur une matière répandue de façon égale à travers les cieux est incompatible avec mon système." 19

Pourtant, c'est précisément contre ce point de vue que s'élève Kant, car il risque de donner libre cours à l'imagination la plus fantaisiste des causes finales:

"La célèbre théorie de Newton ne doit pas servir de prétexte a une confiance paresseuse, ni amener à prendre pour une explication conçue dans un esprit philosophique un recours précipité à une disposition directe de Dieu. [...] On s'expose encore au préjudice consistant à laisser de côté les causes efficientes, et à passer directement à une cause finale purement imaginaire." 20

Bref, Kant reproche à Newton d'avoir suivi la voie de la physico-théologie ordinaire qui considère que tout ordre, toute perfection ou toute harmonie sont purement contingents dans la nature. Ce qui reviendrait à dire que c'est seulement en violentant la nature que Dieu la conduit à produire les effets particuliers qu'il veut y réaliser, comme prétendaient le montrer aussi Burnet ${ }^{21} \mathrm{~W}$. Derham $^{22}$ et W. Whiston ${ }^{23}$ Outre la stérilité intellectuelle de cette "physico-théologie ordinaire" qui rend vaine toute recherche scientifique pour tenter d'expliquer les phénomènes naturels, elle finit par conduire à ce que Kant appelle un "athéisme raffiné", puisqu'elle conduit non pas à un Dieu créateur tirant la matière du néant, mais à un "artisan qui l'ordonne et la façonne"24. D'ailleurs, la

19 Newton, Lettre à Bentley du 11 février 1693, in Newton's Correspondance, III, p. 244.

20 Op. cit., IIe. Partie, Ve. Considération, AK II, p. 121-2; trad. fr., Pléiade, T. I, p. 386-7.

21 Burnet, Telluris theoria sacra, 1681; Kant le critique in L'Unique fondement possible..., 1763, Iie. Partie, Vie. Considération, AK II, p. 127; trad. fr., Pléiade, T. I, p. 393.

22 William Derham, Physico-Theology, London, 1713, Rotterdam 1726, tr. fr. J. Lufnen, Paris, 1730; Astrotheology, London, 1714, trad. fr., Bellanger, Paris, 1729; trad. allemande Fabricius, Hambourg, 1728.

23 William Whiston, Astronomical Principles of Religion, London, 1726. Kant critique Whiston dans une note, cf. op. cit., IIe. Partie, Ve. Considération, AK II, p. 120-121; trad. fr., Pléiade, T. I, p. 385.

24 Op. cit., IIe. Partie, Ve. Considération, AK II, p. 122-3; trad. fr., Pléiade, T. I, p. 388. 
physico-théologie ordinaire mérite, à juste titre selon Kant, les railleries de Voltaire dont il cite l'échantillon suivant: "Voyez bien, si nous avons un nez, c'est assurément pour y pouvoir poser des besicles"25

Certes, toute forme de physico-théologie, quelle qu'elle soit, admet qu'il existe une dépendance <Abbängigkeit> des choses de la nature envers Dieu. Or, cette dépendance peut être soit une connexion contingente, soit une connexion nécessaire. Certains philosophes anciens comme Aristote avaient déjà admis qu'il existe une dépendance entre le Monde et Dieu, mais Kant insiste sur le fait que c'est à la suite des enseignements de la Révélation que cette dépendance a été spécifiée par l'idée biblique de création $^{26}$. Celle-ci implique la position absolue de la chose à la fois dans son essence et dans son existence, dans sa forme et dans sa matière, tandis que la physico-théologie ordinaire considère Dieu comme l'artisan qui façonne l'ordre du Monde par un décret libre et contingent de sa volonté toute-puissante. Il faudra donc que la physico-théologie perfectionnée $<$ verbesserte $>$ puisse conduire à l'existence d'un Dieu créateur.

Toute l'originalité de la physico-théologie perfectionnée $<$ verbesserte $>$ du Bewneisgrund consiste précisément à montrer que l'ordre de la nature possède une unité nécessaire qui seule permet de remonter à l'existence d'un Dieu Créateur. Or, c'est la cosmologie, et mieux encore la cosmogonie, qui permet à Kant de faire ressortir cette unité nécessaire. Est-ce à dire que tout soit absolument nécessaire dans l'ordre du monde?

Tout d'abord, Kant admet que l'existence même des choses naturelles demeure toujours contingente. I/ admet aussi que de très nombreuses connexions soumises à des lois différentes à l'intérieur d'un même être relèvent d'une unité contingente et particulière, bien que nous ignorions ce qui assure l'unité interne des êtres vivants. En revanche, lorsque c'est un même principe qui commande la conformité de différentes choses à des lois qui impliquent à leur tour l'intervention d'autres lois, il règne une unité nécessaire. Kant allègue à titre d'exemple, pour illustrer son propos, le cas du "règne végétal" et du "règne animal" qui comportent une unité contingente remarquablement sage, mais tout de même con-

25 Op. cit., IIe. Partie, VIe. Considération, AK II, p. 131; trad. fr., Pléiade, T. I, p. 398. Cette citation de Voltaire est tirée de son Dictionnaire philosopbique, à l'article: "Fin, causes finales"

26 Op. cit., IIe. Partie, VIe. Considération, AK II, p. 124; trad. fr., Pléiade, T. I, p. 390. "C'est peut-être seulement depuis que la Révélation a enseigné le principe de la dépendance complète du monde à l'égard de Dieu que la philosophie a commencé à faire l'effort nécessaire pour poser le problème de l'origine des choses elles-mêmes, c'est-à-dire de l'existence de la matière brute de la nature, et pour montrer qu'elle est impossible sans un Créateur" 
tingente, car elle est arbitraire. C'est plutôt entre les lois universelles de la "nature inorganique" que l'on découvre une unité nécessaire:

“[...] Ce ne sont pas des causes différentes qui font que la Terre est ronde, que les corps terrestres résistent à la force centrifuge, que la Lune est maintenue sur son orbite. La seule pesanteur est la cause suffisante de la production de ces effets. [...] La nature inorganique apporte [...] des preuves innombrables en faveur d'une unité nécessaire, résultant de la convenance de nombreux effets à un principe simple." 27

Sur ce point, il faut remarquer que Kant suit de très près les vues que Maupertuis avait développées dans son Essai de Cosmologie de 1750 et dont une traduction allemande vit le jour un an plus tard ${ }^{28}$. Tout comme lui, Kant critique et rejette les physicothéologies ordinaires qui voient dans chaque effet naturel le résultat d'un miracle indispensable pour réaliser les desseins de la Providence divine. Tout comme lui aussi, Kant propose de promouvoir une physicothéologie rénovée qui intègre les résultats les plus récents des sciences de la nature $^{29}$ Enfin et surtout, Kant pense pouvoir s’appuyer sur un principe général qui unifie les diverses lois du mouvement ${ }^{30}$ : le principe de 1 a moindre action de Maupertuis, qui ser d'unité nécessaire aux lois du mouvement:

"M. de Maupertuis a démontré que même les lois les plus générales $[. .$.$] obéissent toutes à une règIe dominante d'après$ laquelle est observée, dans l'action, la plus grande économie Par cette découverte, les effets de la matière, en dépit des grandes diversités qu'ils peuvent présenter entre eux, sont ramenés à une

27 Op. cit., Ile. Partie, IIIe. Considération, AK II, p. 106-7; trad. fr., Pléiade, T. I, p. 368-9.

28 Maupertuis, Essai de Cosmologie, 1ère. éd. française, Berlin, 1750; trad. allemande, Versuch einer Cosmologie, Berlin, 1751.

29 De même, Maupertuis remarquait dans son Essai de Cosmologie, éd. fr. de 1751, Avant-Propos, p. 55: "Ce n'est donc point dans les petits détails, dans ces parties de l'Univers dont nous connoissons trop peu les rapports, qu'il faut chercher l'Être suprème: c'est dans les phénomènes dont l'universalité ne souffre aucune exception, \& que leur simplicité expose entièrement à notre vûe"

30 Maupertuis écrivait dans l'Essai de Cosmologie, éd. fr. de 1751, Avertissement, p. xix: "J'ai découvert un principe métaphysique sur lequel toutes les lois du mouvement et du repos sont fondées. J'ai fait voir la conformité de ce principe avec la puissance \& la sagesse du créateur \& de l'ordonnateur des choses". 
formule générale qui exprime une aptitude à la convenance <Anständigkeit>, à la beauté et à l'harmonie <Wohlgereimtheit> [...] C'est avec raison qu'il a cru que [...] un accord si universel dans les natures les plus simples des choses met à notre disposition une raison suffisante pour trouver avec certitude, dans quelque Être originaire et parfait la dernière cause de tout dans l'univers. ${ }^{31}$

L'avantage énorme que présente le principe maupertuisien de la moindre action, c'est non seulement qu'il ramène la diversité des lois du mouvement à l'unité d'un principe, mais aussi qu'il permet de réconcilier le mécanisme avec la finalité: tel est bien le sens de ce que Kant entend sous le terme de convenance $^{32}$ $<$ Anständigkeit> selon lequel les effets ne sont pas seulement des résultats, mais aussi des fins. Ainsi, Kant pense pouvoir tirer la physico-théologie de l'impasse dans laquelle Newton l'avait entrainée lorsqu'il s'était penché sur le problème cosmologique. Au lieu de recourir, comme Newton, à la théologie et aux causes finales pour rendre raison de la stabilité et de l'ordre cosmique, Kant prend appui sur l'unité et la convenance des lois universelles pour montrer qu'entre le fiat créateur de Dieu et l'ordre cosmique que découvre l'astronomie, "les lois mécaniques ont une prédisposition à l'ordre"33 qui a dû permettre d'organiser l'état originellement chaotique de la matière. Kant pense avoir ainsi épuré la physicothéologie des scories d'un finalisme incontrôlé et avoir donc permis au newtonianisme de réintégrer le problème cosmologique dans le cadre de la mécanique rationnelle:

"Si [Newton] avait pu former une hypothèse bien fondée sur la constitution primitive de la matière, on peut même être certain qu'il eût cherché, selon le procédé requis par la philosophie, les principes de la constitution de l'univers < die Gründe von der Beschaffenbeit des Weltbaues $>$ dans les lois générales de la mécanique, sans craindre pour cela que cette explication de l'ori-

31 Op. cit., IIe. Partie, Ie. Considération, AK II, p. 98-99; trad. fr., Pléiade, T. I, p. 357-8.

32 On lit également dans l'Essai de Cosmologie de Maupertuis, éd. fr. de 1751, Avertissement, p. xvii: “'C'est ainsi que malgré quelques parties de l'univers dans lesquelles on n'aperçoit pas bien l'ordre et la convenance, le tout en présente assez pour qu'on ne puisse douter de l'existence d'un Créateur tout-puissant \& tout sage"

33 Op. cit., IIe. Partie, VIe. Considération, AK II, p. 130; trad. fr., Pléiade, T. I, p. 396. 
gine du monde fasse passer la création des mains de Dieu à la puissance du hasard." 34

Bref, le jeune Kant du Beweisgrund pense avoir engagé la cosmologie dans la voie sûre de la science newtonienne. Examinons quels sont les traits saillants de son hypothèse cosmogonique qui ne se présente plus sous le titre arrogant de Théorie du ciel.

\section{Les remaniements de l'hypothèse cosmologique de 1755 dans le Beweisgrund}

Le titre de la VIIe. Considération est: Cosmogonie. Il ne fait que reprendre le titre que le jeune Kant avait voulu donner originellement à sa Théorie du ciel lors de l'annonce de sa parution dès 1754 dans un petit Mémoire destiné à l'Académie des Sciences de Berlin. Le parallèle est frappant:

1754

Kosmogonie, oder Versuch, den Ursprung des Weltgebäudes, die Bilder Himmelskörper und die Ursachen ihrer Bewegung aus den allgemeinen Bewegungsgesetzen der Materie der Theorie des Newtons gemäB ber qu leiten 36 .
1763

Kosmogonie

Eine Hypothese mecanischer Erdung klärungsart des Ursprungs der Weltkörper und der Ursachen ihrer Bewegungen, gemäB denen vorher erwiesen Regeln ${ }^{35}$.

On notera au passage à quel point Kant tient à ses recherches cosmologiques antérieures, puisque près de dix années n'ont rien changé à sa détermination intellectuelle.

La petite introduction qui précède l'hypothèse et qui lui sert d'avertissement, expose les données du problème. Celui-ci consiste à expliquer la formation des corps célestes ainsi que leurs mouvements à l'aide de la force fonda-

34 Op. cit., IIe. Partie, Ve. Considération, AK II, p. 121; trad. fr., Pléiade, T. I, p. 386.

35 Op. cit., IIe. Partie, VIIe. Considération, AK II, p. 137; trad. fr., Pléiade, T. I, p. 405.

36 Le titre de ce petit Mémoire de 1754 était: Recberches sur la question: la Terre a-t-elle subi quelques modifications dans son mouvement de rotation axiale depuis son origine?, cf. AK I, p. 190-1. 
mentale <eine Grundkraft der Materie> d'attraction dont on ne peut ni ne doit expliquer l'existence puisqu'elle est originellement constitutive de la matière. Sur ce point, Kant se montre infidèle à la pensée de Newton qui avait toujours refusé, pour sa part, de considérer l'attraction comme une force essentielle aux corps $^{37}$ Il reste donc à rendre compte d'une part du "mouvement d'impulsion" $<$ Wurfsbewegung, Schwung $>$ et d'autre part de sa "direction" $<$ Ricbtung $>$ qui s'ajoutent aux effets de l'attraction dans la constitution du système du monde. Face au préjugé qui objecterait qu'il est téméraire d'essayer d'expliquer l'origine du système du monde alors que l'on est incapable d'expliquer "un vulgaire brin d'herbe", Kant répond par le fait du succès incontestable de la mécanique dans le monde inorganique explicable en raison de sa grande simplicité, tandis que l'incroyable et admirable complexité du monde organique le rend pour l'époque inaccessible.

Le plan de la Cosmogonie de 1763 est identique à celui de la Théorie du ciel, $\grave{a}$ cette seule différence près que les considérations sur les habitants des planètes (qui figuraient à la IIIe. Partie du livre de 1755) ont été purement et simplement supprimées. En fait, dans une première section, Kant examine l'actuelle structure d'ensemble de l'univers, en développant sa théorie de la Voie lactée et des "Univers-îles"; puis, dans les trois autres sections, il esquisse la formation mécanique de tout système planétaire, avant de proposer une nouvelle estimation de la durée de la rotation axiale de Saturne.

\section{II.1. l'actuelle structure d'ensemble de l'Univers}

Kant n'a rien changé dans son hypothèse, si ce n'est la forme de son exposé qui est plus pure et totalement dépourvue de digression sur les autres hypothèses en vigueur. Kant suit le fil de l'analogie en partant du système solaire et en s'élevant successivement à la Voie lactée (qui est un système de systèmes solaires), puis aux autres voies lactées que doivent être les nébuleuses récemment observées depuis un siècle (mais dont il affirme sans preuve la situation extragalactique), et il arrive enfin aux éventuels amas galactiques qui sont des systèmes d'ordre 3 , tout en laissant ouverte la question de savoir s'il existe

37 Isaac Newton, Principia mathematica Pbilosopbiae Naturalis, Livre III, Regulae pbilosophandi, trad. fr. de la Marquise du Chatêlet, 1759, réed. Blanchard, 1966, T. 2, p. 4: “Cependant, je n'affirme point que la gravité soit essentielle aux corps. Et je n'entends par la force qui réside dans le corps, que la seule force d'inertie, laquelle est immuable; au lieu que la gravité diminue lorsqu'on s'éloigne de la Terre". 
encore des systèmes supérieurs. Tous ces ordres, tous ces systèmes sont régis par les mêmes lois et c'est la raison pour laquelle ils doivent présenter la même structure à des échelles différentes:

"Cette analogie fournit ici une raison sérieuse de supposer: [...] que les soleils, au nombre desquels figure le nôtre,, constituent un système du monde réglé selon des lois semblables à celles de notre système planétaire, en petit." 38

Kant sait pertinemment que sa conception n'est pas communément reçue dans le monde savant, mais, en nous renvoyant au catalogue d'opinions que Maupertuis dresse à ce sujet dans son Discours sur les différentes figures des astres 39 il montre bien qu'il n'en a cure. Ce qui est nouveau, dans la version de 1763, c'est que Kant n'affirme plus que cet emboîtement de systèmes de plus en plus vastes s'étend à l'infinin. Il ne soulève même plus la question ${ }^{40}$ Nous aurons l'occasion d'y revenir. Toutefois, étant donné que cette théorie des "univers-îles" est purement conjecturale, Kant encourage les recherches observationnelles sur les "nébuleuses" pour mettre à l'épreuve son hypothèse dans un avenir assez proche.

\section{II.2. La formation mécanique du système planétaire}

Avant d'exposer directement son hypothèse cosmogonique, Kant éprouve le besoin de plaider en faveur d'une explication d'ordre mécanique, sans

38 Op. cit., IIe. Partie, VIIe. Considération, AK II, p. 140; trad. fr., Pléiade, T. I, p. 409.

39 Op. cit., IIe. Partie, VIIe. Considération, AK II, p. 141; trad. fr., Pléiade, T. I, p. 410: “L'astronomie nous a découvert depuis longtemps de telles petites taches, malgré la diversité des opinions qu'on a à leur sujet, comme on peut le voir d'après les oeuvres de Maupertuis sur les figures des astres" Kant ne connait qu'un resumé, paru dans les Acta Eruditorum en 1745, de l'ouvrage de Maupertuis intitulé Discours sur les différents figures des astres et qui avait été publié pour la première fois à Paris en 1732, la seconde édition date d 1742. Dans l'édition de 1768, Maupertuis mentionne seulement les observations et les interpretations de Huygens, Halley, W. Derham, mais il pense que sa propre interprétation est la seule qui soit juste, cf. in Oeuvres de Maupertuis, Lyon, 1768, réed. Olms, 1974, T. I, p. 145: "Tous ces phénomènes, dit-il, se trouvent par notre système si naturellement \& si facilement expliqués, qu'il n'est presque pas besoin d'en faire l'application"

40 Comparer avec la Théorie du ciel, Ière partie, AK I, p. 256: Nous voyons les premiers termes d'une progression continue de mondes et de systèmes, et cette première partie d'une progression infinie <unendlicbe Progression> nous donne déjà à réconnaitre ce qu'ilfaut conjecturer de l'ensemble. Cette série n'a pas de fin, elle s'enfonce dans un abîme véritablement insondable. 
invoquer le recours aux causes finales comme le fit Newton. Contre la physicothéologie ordinaire qui se réfugie dans l'intervention directe de Dieu, Kant montre que si l'ordre du système solaire est mathématisable et conforme aux lois physiques générales, il n'est pas pour autant absolument parfait: il connait des écarts $<$ Abweicbungen $>$, des dérèglements $<$ Regellosigkeit $>$ et des aberrations, Or, ces écarts vis-à-vis de la perfection $<$ Vollkommenheit $>$ (a savoir que les corps célestes ne sont pas des sphères parfaites, que les orbites planétaires ne sont pas parfaitement circulaires, que les plans des orbites s'écartent légèrement de celui de l'équateur solaire, pour ne rien dire des comètes), tous ces écarts prouvent donc que l'ordre cosmique n'est pas causé directement par Dieu, mais par l'intermédiaire des lois générales de la mécanique:

"Dans une ouvre immédiatement divine, il ne peut jamais, au contraire, y avoir, de place pour des fins incomplètement atteintes." 41

La voie est donc libre pour exposer tout d'abord les grandes lignes de la formation du Soleil à partir de la matière primitive <aus dem Grundstoffe > qui était répandue à travers l'espace cosmique dans un état de diffusion extrême. Le jeu de la force d'attraction condense autour du point de plus forte attraction toutes les particules qui chutent sur ce qui deviendra peu à peu le Soleil, tout en vidant progressivement l'espace.

Kant pense pouvoir expliquer la rotation de ce proto-soleil par la chute des particules animées d'un mouvement tangentiel dû aux chocs entre les particules lors de leur chute convergente vers le centre de gravité du Soleil. Dans un second moment, le reste des particules, qui étaient assez éloignées du Soleil et dotées d'un mouvement circulaire suffisamment rapide pour contrebalancer les effets de són attraction, continuent d'orbiter autour du Soleil en se regroupant dans le plan de l'équateur solaire ${ }^{42}$. Peu à peu les points de plus forte attraction permettent aux proto-planètes de se former par un mouvement de condensation qui les conglobe progressivement, tout en éloignant d'autant plus du Soleil ces proto-planètes qu'elles sont moins denses.

On est frappé de voir que dans le Bewweisgrund, tout comme dans la Théorie du ciel, Kant ne se donne pas la peine de chiffrer les vitesses, les distances, les masses, les densites spécifiques des corps célestes en formation, alors qu'il pré-

41 Op. cit., Ile. Partie, VIIe. Considération, AK II, p. 144; trad. fr., Pléiade, T. I, p. 413.

42 Op. cit., IIe. Partie, VIIe. Considération, AK II, p. 146-147; trad. fr., Pléiade, T. I, p. 417. 
tend être "en mesure d'indiquer la condition qui, seule, rend possible une explication mécanique des mouvements célestes" 43 Kant est conscient du caractère très grossier et approximatif de son esquisse et il invite "des hommes d'esprit large à un examen plus approfondi" 44 Il n'exclut pas que l'on puisse même découvrir d'autres hypothèses mécaniques, miieux conçues que la sienne, car ce qui le préoccupe avant tout, c'est d'avoir établi définitivement qu'une explication mécanique de la formation de l'univers est "compatible avec la foi en un Dieu sage" 45

Malgré ce dédain pour le calcul et la mesure, Kant consacre environ un quart de son exposé pour expliquer sa méthode de calcul de la durée de la rotation de Saturne, sans montrer pour autant ses propres calculs dont il nous confie seulement le résultat final. Malheureusement, il aboutit à un résultat différent de celui qu'il obtint en 1755: 5h. 40' en 1763 et 6h. 23' 53" en $1755 .{ }^{46}$ Cette

43 Op. cit., IIe. Partie, VIIe. Considération, AK II, p. 145; trad. fr., Pléiade, T. I, p. 415.

44 Op. cit., IIe. Partie, VIIe. Considération, AK II, p. 148; trad. fr., Pléiade, T. I, p. 419.

45 Ibidem.

46 La loi sur laquelle repose ce calcul est un genre de réplique de la 3e. loi de Kepler faisant entrer en jeu l'accélération centrale du mouvement circulaire qui est en raison directe du carré de la vitesse, mais inverse de la distance au centre $=\mathrm{v}^{2} / \mathrm{r}$. Kant réduit cette formule à $\frac{\mathrm{V}}{\sqrt{\mathrm{r}}}$, puisqu'il écrit in

AK I, p. 294: “on peut trouver la vitesse de la rotation axiale de Saturne en la déduisant de la vitesse d'un des satellites de Saturne, [ici, le 4e. c'est-à-dire Titan découvert par Huygens en 1655] du fait qu'on trouve cette vitesse dans le rapport des racines carrées des distances au centre de la planète". Kant établit les rélations suivantes au niveau de la force centrifuge: $\gamma=\omega^{2} \mathrm{R}$ et comme $\omega=\frac{2 \pi}{\mathrm{T}}$ on a $\gamma=4 \pi^{2}\left(\frac{\mathrm{R}}{\mathrm{T}^{2}}\right)$ Si on néglige $4 \pi^{2}$, il reste $\left(\frac{\mathrm{R}}{\mathrm{T}^{2}}\right)$ ou $\frac{\sqrt{\mathrm{R}}}{\mathrm{T}}$ Si l'on met en rélation ces deux formules on obtient: $\frac{\mathrm{v}}{\sqrt{\mathrm{R}}}=\frac{\sqrt{\mathrm{R}}}{\mathrm{T}} \quad$ d'où l'on tire $R=T . \mathrm{T}$ ou même

$T=\frac{R}{V}$ Il ne reste plus qu'à mettre en forme la comparaison des temps, des rayons et des vitesses respectivement du satellite Titan (paramètres connus grâce à l'observation), avec les rayons'de Saturne (rayon équatorial) et du bord interne de l'anneau (connus et mésurés par Cassini et repris par Newton in Principia, III, p. 7 e 8 du vol. II de la traduction de la Marquise du Chatelet). Une dernière précaution d'écriture consistera à se débarasser de la vitesse encore inconnue du bord interne de l'anneau par la rélation suivante où $v_{1}$ et $\sqrt{R_{1}}$ désignent respećtivement la vitesse et la racine du rayon du sattelite, et $\mathrm{v}_{2}$ et $\sqrt{\mathrm{R}_{2}}$ celles du bord intérieur de l'anneau: $\frac{\sqrt{\mathrm{R}_{2}}}{\sqrt{\mathrm{R}_{1}}}=\frac{\mathrm{v}_{1}}{\mathrm{v}_{2}}$; d'où l'on tire $\mathrm{v}_{2}=\frac{\mathrm{v}_{1} \cdot \sqrt{\mathrm{R}}}{\sqrt{\mathrm{R}_{2}}}$.Si l'on réprend les indications de Gensichen données dans l'édition de 1791, et si l'on garde notre type de notation en ajoutant $r$ pour désigner le rayon de Saturne et $(T, t)$ respectivement pour le temps de révolution du satellite et pour le temps de rotation axiale de Sa- 


\section{Le retrait de l'infinitisme en cosmologie et son statut théologique}

Bien que Kant reprenne, sous une forme abrégée, l'essentiel de sa Théorie $d u$ ciel, il est tout à fait manifeste que la différence fondamentale avec cette dernière c'est que le Beweisgrund ne dit plus un seul mot sur l'infinité de l'univers. Ce n'est qu'à la Considération qui fait suite à l'exposé de sa Cosmogonie (Huitième Considération) que Kant revient sur l'idée d'infini, et notamment sur l'infinité divine, mais c'est pour prendre ses distances avec ce terme dont il nous dit qu'en plus de ses déterminations conceptuelles propres, il possède une charge émotionnelle.

"Certes le terme infini est beau et proprement esthétique. Ce qui dépasse tous les concepts du nombre émeut et met l'âme dans un état d'étonnement, en la troublant en quelque sorte. ${ }^{\text {50 }}$

Kant se laisse pourtant aller à quelques débordements enthousiastes dans ce livre, comme dans l'extrait suivant, mais en note seulement:

"Quand j'élève mes yeux vers le haut pour regarder l'espace immense <unermeBlichen Raum> où pullulent des mondes comme s'ils étaient des poussières, aucune langue humaine n'est capable d'exprimer le sentiment que suscite en moi une telle pensée, et la plus subtile des analyses métaphysiques ne résiste pas à la sublimité et à la noblesse d'une pareille contemplation $<$ Anschauung $>$." 51

Tout en démontrant ainsi son profond attachement à l'idée d'infini, Kant la chasse du domaine de la cosmologie et lui donne même momentanément congé dans la mesure où il considère que le termé d"“omnisuffisance $<$ Allgenugsamkeit >> de Dieu "fournit pour désigner la plus grande perfection de cet Etre un terme beaucoup plus juste que celui de l'Infini dont on se sert ordinairement'52. Kant précise que l'emploi du terme infini, qui s'applique aussi bien aux mathématiques qu'à Dieu et aux choses créées, risque d'introduire toutes sortes d'équivo-

50 Kant, L'Unique fondement possible..., 1763, AK II, p. 154: trad. fr. Zac, Pléiade, T. I, p. 426.

51 Op. cit., Ile. Partie, Ve. Considération, AK II, p. 117; trad. fr., Pléiade, T. I, p. 381. Traduction rectifiée par nos soins.

52 Op. cit., IIe. Partie, VIIIe. Considération, AK II, p. 154; trad. fr., Pléiade, T. I, p. 426. 
ques et de confusions dont la pire est sans doute de constituer, "entre de telles déterminations de Dieu et celles des choses créées, une analogie bien difficile à établir"53. Selon Kant, le plus grand danger qui menace l'infinitisme cosmologique, c'est une éventuelle collusion entre l'infinité divine et l'infinité cosmique dans une sorte de monisme réducteur. On sent ici, chez le Kant de l'Unique fondement, le souci d'éviter toute déviance possible vers ce qui pourrait être taxé de panthéisme, voire de matérialisme ou d'athéisme, et surtout de spinozisme. Il lui faut montrer au contraire que l'unique fondement possible d'une preuve de l'existence de Dieu débouche sur un Dieu transcendant, immatériel et "allgenugsam" C'est pourquoi il écrit dans le Beweisgrund afin de laver son traité de toute accusation possible de spinozisme:

“Le monde n'est pas un accident de la divinité, puisqu'on y trouve des conflits, des manques et des changements, déterminations contraires à celles de la divinité. Dieu n'est pas la substance unique que existe; et toutes les autres choses sont aussi des substances, mais des substances dépendantes." 54

Il faut rappeler que le Beweisgrund est le seul texte précritique avant 1770 où Kant se livre à une critique du spinozisme en citant même le nom de Spinoza ${ }^{55}$ Bien avant que n'éclate le Pantheismusstreit, le spinozisme faisait déjà horreur à la plupart des penseurs du XVIIIe. siècle au point de servir d'invective officielle dans la plupart des querelles intellectuelles, même si le spinozisme demeurait totalement incompris et seulement perçu à travers l'article célèbre de Bayle dans son Dictionnaire bistorique et critique ${ }^{56}$ Kant ne pouvait ignorer cette grave accusation même pendant ses années d'étude à l'Université de Königsberg, puisqu'elle avait opposé les Piétistes anti-wolffiens à l'Ecole de Wolff ${ }^{57}$ durant toute sa jeunesse. A l'époque de la rédaction de sa Théorie du ciel, le style

\section{Ibidem.}

54 Op. cit., Ie. Partie, IVe. Considération, AK II, p. 90-1; trad. fr., Pléiade, T. I, p. 349.

55 Op. cit., Ie. Partie, Ie. Considération, AK II, p. 74; trad. fr., Pléiade, T. I, p. 328: "Le Dieu de Spinoza est soumis à de perpétueles changements"

56 Bayle, Dictionnaire historique et critique, Rotterdam, 1697 et 1702, T. III, article "Spinoza": trad. allemande par Gottsched (1741-1744).

57 Dans la Luculenta commentatio, Wolff s'efforce de montrer que si son système admet l'idée d'un progressus in infinitum, il ne conduit nullement au spinozisme ni à l'athéisme pour autant, contrairement à ce que prétendaient les Piétistes violemment anti-wolffiens. C'est pourquoi Wolff n'admettait qu'un infini potentiel pour la série des êtres créés finis et il réservait à Dieu seul l'infinité actuelle 
du jeune Kant était celui d'un métaphysicien intempérant qui ne reculait pas devant le danger d'accusation de "spinozisme", c'est certainement ce qui avait dû le conduire à publier sa cosmologie sans nom d'auteur. Aussi, il n'avait pas hésité à recourir à l'argument de type théo-cosmologique pour déduire l'infinité cosmique à partir de l'infinité divine en écrivant:

"La conception que nous avons exposée nous ouvre une perspective dans le champ infini de la Création <eine Aussicbt in das unendliche Feld der Schöpfung $>$ et offre une représentation $<$ Vorstellung $>$ de l'oeuvre de Dieu $<$ dem Werke Gottes $>$ conforme à l'infinité du grand Maître d'œuvre < der Unendlicbkeit des groBen Werkmeisters $>$ [...] Il n'y a pas ici de fin, mais un abîme d'une veritable immensité $<$ UnerneBlicbkeit $>$ dans lequel sombre tout le pouvoir des concepts humains. celui-ci fut-il rendu supérieur grâce à la science des nombres. La Sagesse, la Bonté, la Puissance qui sont manifestées <offenbart> sont infinies <unendlicb> et dans la même mesure fécondés et actives, le plan de leur manifestation doit donc être comme elles infini <unendlich> et sans limites." 58

Cet argument théo-cosmologique qui remonte pour l'essentiel à Bruno $^{59}$, (et dont Kant a dû avoir connaissance au moins en parcourant $l$ Histoire critique de la Pbilosopbie de Brucker $\left.{ }^{60}\right)$, ne réapparaîtra plus jamais, du moins sous cette forme, dans l'oeuvre de Kant. A l'époque du Beweisgrund Kant ne peut donc

qu'il refuse d'attribuer à l'univers. Cf. Wolff, De differentia nexus rerum sapientis et fatalis necessitatis, nec non systematis barmoniae praestabilitae et bypothesium Spinosae luculenta commentatio, Halle, 1723 et 1737, sect. I, $₫ 16$, p. 52-3.

58 Cf. Kant, Théorie du ciel, Ière partie, AK I, p. 255-6; trad. f. Roviello, Paris, Vrin, 1984, p. 96-7.

59 Par exemple, cf. Bruno, Cena de le Ceneri, 1584, trad. fr. Y. Hersant, Premier Dialogue, éd. De l'Eclat, 1988, p. 26: "Ainsi sommes-nous conduits à découvrir l'effet infini de la cause infinie, la trace vivante et véritable de la vigueur infini" Cf. aussi: Bruno, De l'Infinito, 1584, trad. fr. Levergeois, Berg. 1987, Dialogue Premier, p. 69: "Qui nie l'effet infini, nie la puissance infinie", Bruno, Documenti veneti, XI, Interrogatoire du 2 juin 1592, in Vita di Giordano Bruno con documenti editi e inediti, V. Spampanato, Messina, 1921, rééd. par Nuccio Ordine, Gela Editrici, Roma, 1988, p. 709: “[...] perché io stimavo cosa indeǵna della divina bontá e potenza che, possendo produr oltra questo mondo un altro ed altri infiniti, producesse un mondo finito"

60 Cf. Jacob Brucker, Historia critica philosopbiae a mundi incunabulis ad nostram usque aetatem deducta, Leipzig, 1742-1744 (1ère éd.), 5 vols., spécialement le tome IV. Brucker avait finement montré que la philoșophie de Bruno qu'il connaissait bien (pour avoir étudié de très près le De la Causa et le $D e$ Immenso et innumerabilibus) différait profondément de celle de Șinoza, contrairement à ce qu'en 
plus se permettre de recourir à l'argument de type théo-cosmologique qui repose sur l'infinité divine, car il se méfie du concept d'infini en général, même en théologie où il préfère employer le terme d'A1 lgenugsamkeit à propos de Dieu. C'est d'ailleurs la raison pour laquelle il n'hésitait pas à qualifier certaines ces hypothèses de la Théorie du ciel d'“assez risquées"61 dans l'Avant-propos du Bewveisgrund. Désormais, Kant s'exprime officiellement et ne peut plus prendre autant de risques qu'auparavant. Pourtant, il sera aussitôt attaqué, dès la publication de son Bewveisgrund, pour son spinozisme larvé. C'est ce que prétend l'attaque qui a été dirigée directement contre le Beweisgrund par un jeune philosophe allemand, Daniel Weymann, auteur d'une plaquette intitulée Bedenklicbkeiten über den einzig möglichen Beweisgrund des Herrn I. Kants zu einer Demonstration des Daseyns Gottes $^{62}$ Weymann reproche à la démonstration alléguée par Kant d'aboutir à un Dieu tout à fait semblable à celui de la substance unique de Spinoza. Comme on le sait, Kant ne répondra aux accusations de spinozisme pas avant la mort de son ami M. Mendelssohn en 1786 à l'occasion du déchaînement du Pantheismusstreit. Certes cette querelle du panthéisme sort du cadre de notre communication sur la cosmogonie de 1763, puisqu'elle a lieu au cours de la période critique. Mais il n'est pas inutile de rappeler que ce qui a permis à Jacobi d'accuser le grand Kant de spinozisme, c'est sa conception de l'espace infini dans la première Critique. Kant avait donc bien fait de laisser de côté la question de l'infinité cosmique dès 1763 , car elle risquait de l'entraîner dans des polémiques stériles, dans la mesure où elles l'écartent de son but essentiel: sortir la métaphysique de l'impasse où elle était arrivée au XVIIIe. siècle. Les difficultés de l'infini seront le pivot central des deux premières Antinomies de la raison pure, mais cette fois au profit de l'entreprise critique de Kant.

avait écrit Bayle dans son Dictionnaire bistorique et critique. Peut-être le jeune Kant avait-il entendu parler de Bruno par le canal de l'Ecole wolffienne à propos de la querelle qui opposa entre 1720 et 1730 Joachim Lange à Wolff à propos des rapports entre foi et raison et de l'accusation de spinozisme. Toutefois, il ne s'agit là que de suppositions extrêmement ténues qui ne reposent sur aucun document précis, ni sur aucun témoignage direct. On peut consulter utilement sur cette question délicate les indications générales qui figurent dans les articles suivants: Jean-Louis Vieillard-Baron, Bruno et l'idéalisme allemand; RMM, 1971, 76, p. 406-423; Saverio Ricci, La ricezione del pensiero do Giordano Bruno in Francia e in Germania da Diderot a Schelling, Giornale Critico della Filosofia Italiana, Firenze, Fasc. III, Settembre-Dicembre 1991, p. 431-465.

61 Kant, L'Unique fondement possible..., 1763, AK II, p. 69; trad. fr. Zac, Paris, T. I, Avant-propos, p. 322, "mit verschiedenen etwas gewagten Hypothesen".

62 Daniel Weymann, Bedenklichkeiten über den einzig möglichen Beweisgrund des Herrn I. Kants zu einer Demonstration des Daseyns Gottes, Königsberg, 17.63, chez J.J. Kanter, le même éditeur que Kant. 